\title{
Age-Related Differences in the Survival Benefit of Anticoagulant Therapy in Sepsis in Accordance with the Japanese Association for Acute Medicine Disseminated Intravascular Coagulation Diagnostic Criteria: A Retrospective Sub-analysis of a Prospective Multicenter Study
}

Takeshi Wada ( $\sim$ twada1@med.hokudai.ac.jp )

Hokkaido University Faculty of Medicine https://orcid.org/0000-0001-5220-3342

Kazuma Yamakawa

Osaka Medical College: Osaka Ika Yakka Daigaku

Daijiro Kabata

Osaka City University: Osaka Shiritsu Daigaku

Toshikazu Abe

University of Tsukuba: Tsukuba Daigaku

Hiroshi Ogura

Osaka University: Osaka Daigaku

Atsushi Shiraishi

Kameda Medical Center

Daizoh Saitoh

National Defense Medical College: Boei Ika Daigakko

Shigeki Kushimoto

Tohoku University: Tohoku Daigaku

Seitaro Fujishima

Keio University School of Medicine Graduate School of Medicine: Keio Gijuku Daigaku Igakubu

Daigakuin Igaku Kenkyuka

Toshihiko Mayumi

University of Occupational and Environmental Health Japan: Sangyo Ika Daigaku

Toru Hifumi

St Luke's Hospital

Yasukazu Shiino

Kawasaki Medical School: Kawasaki lka Daigaku

Taka-aki Nakada

Chiba University: Chiba Daigaku 


\section{Takehiko Tarui}

Kyorin University: Kyorin Daigaku

\section{Yasuhiro Otomo}

Tokyo Medical and Dental University: Tokyo Ika Shika Daigaku

\section{Kohji Okamoto}

Kitakyushu Municipal Yahata Hospital: Kitakyushu Shiritsu Yahata Byoin

\section{Yutaka Umemura}

Osaka General Medical Center: Osaka Kyuseiki Sogo Iryo Center

\section{Joji Kotani}

Kobe University: Kobe Daigaku

\section{Yuichiro Sakamoto}

Saga University: Saga Daigaku

\section{Junichi Sasaki}

Keio University: Keio Gijuku Daigaku

\section{Shin-ichiro Shiraishi}

\section{Aizu Chuo Hospital}

\section{Kiyotsugu Takuma}

Kawasaki Municipal Hospital: Kawasaki Shiritsu Kawasaki Byoin

\section{Ryosuke Tsuruta}

Yamaguchi University: Yamaguchi Daigaku

\section{Akiyoshi Hagiwara}

National Center for Global Health and Medicine: Kokuritsu Kenkyu Kaihatsu Hojin Kokuritsu Kokusai Iryo Kenkyu Center

\section{Tomohiko Masuno}

Nippon Medical School: Nihon Ika Daigaku

\section{Naoshi Takeyama}

Aichi Medical University: Aichi Ika Daigaku

\section{Norio Yamashita}

Kurume University: Kurume Daigaku

\section{Hiroto Ikeda}

Teikyo University: Teikyo Daigaku

\section{Masashi Ueyama}

Chukyo Hospital

\section{Satoshi Fujimi}

Osaka General Medical Center: Osaka Kyuseiki Sogo Iryo Center

\section{Satoshi Gando}

Sapporo Higashi Tokusyukai Hospital

\section{Research}


Keywords: age, anticoagulants, antithrombin, disseminated intravascular coagulation, sepsis, recombinant human thrombomodulin

Posted Date: June 28th, 2021

DOI: https://doi.org/10.21203/rs.3.rs-626101/v1

License: (c) (1) This work is licensed under a Creative Commons Attribution 4.0 International License. Read Full License 


\section{Abstract}

Background: Disseminated intravascular coagulation (DIC) is one of the major organ dysfunctions associated with sepsis. This study aimed to investigate the age-related differences in the survival benefit of anticoagulant therapy in sepsis in accordance with the DIC diagnostic criteria.

Methods: We conducted a retrospective sub-analysis of a prospective multicenter study. Fifty-nine intensive care units in Japan, from January 2016 to March 2017 were included. Adult patients with severe sepsis based on the Sepsis-2 criteria were enrolled and divided into two groups as follows: anticoagulant group; patients who received anticoagulant therapy, and non-anticoagulant group; patients who did not receive anticoagulant therapy. Patients in anticoagulant therapy group were administered antithrombin, recombinant human thrombomodulin and their combination.

Results: The multivariate Cox proportional hazard regression model including a three-way interaction term among anticoagulant therapy, DIC score and age showed that the increases in the risk were suppressed in patients receiving anticoagulant therapy in patients aged 60 to 70 years with high DIC scores. For patients aged 50 years, the risk in the non-anticoagulant group tended to increase concomitantly with increases in the DIC score in the low score range, while there was no increase in the risk in the high score range and favorable association of anti-coagulant therapy on hospital mortality was not found. In patients aged 80 years, the non-anticoagulant group indicated a certain risk regardless of the DIC score and the anti-coagulant therapy showed no beneficial effect on the decrease in risk of hospital mortality. Furthermore, anticoagulant therapy in the lower DIC score range increased the risk in patients aged 50 to 60 years.

Conclusions: Anticoagulant therapy, the administration of antithrombin, recombinant human thrombomodulin, and their combination, were associated with decreased hospital mortality according to a higher DIC score in septic patients aged 60 to 70 years. Anticoagulant therapy, however, was not associated with a better outcome in relatively younger and older septic patients.

Trial registration: UMIN-CTR, UMIN000019588. Registered on 16 November 2015.

\section{Background}

Disseminated intravascular coagulation (DIC) is characterized by systemic thrombin generation not restricted to the site of insult followed by microvascular fibrin thrombosis. Since the 1990s, DIC is known as one of the major organ failures associated with sepsis [1, 2]. Moreover, DIC gives rise to multiple organ dysfunction and affects patient outcomes [3]. Large-scale randomized clinical trials (RCTs) have been conducted to verify the effects of anticoagulant agents, including the HETRASE study for unfractionated heparin [4], the KyberSept trial for antithrombin [5], the PROWESS, ADDRESS, and PROWESS-SHOCK trials for activated protein C [6-8], the OPTIMIST, and CAPTIVATE trials for tissue factor pathway inhibitor [9, 10] and SCARLET trial for recombinant human thrombomodulin [11]. However, all these studies have failed to determine the efficacy of anticoagulant therapy against sepsis. These results may be associated 
with the fact that most of the RCTs mentioned above targeted heterogeneous patients, such as those with "sepsis" or "severe sepsis" [12]. Recently, it has been widely known that the population fulfilling three factors of "sepsis" and "DIC" and "high disease severity" might be an optimal target for anticoagulant therapies [13]. Our previous study has demonstrated that anticoagulant therapy is associated with better outcome according to the deterioration of both DIC and disease severity simultaneously [14], which were evaluated by the International Society of Thrombosis \& Haemostasis (ISTH) overt DIC scoring system [1] and the Acute Physiology and Chronic Health Evaluation (APACHE) II score [15].

The ISTH overt DIC diagnostic criteria have been known to provide sufficient accuracy in the diagnosis of DIC. However, it has been pointed out that by the time ISTH overt DIC can be identified, the patient might already be at an irreversible and decompensated stage. Thus, it may be too late from therapeutic perspective [3]. The Japanese Association for Acute Medicine (JAAM) DIC diagnostic criteria, which were established to overcome the obstacles of the ISTH overt DIC criteria, proved to have good diagnostic properties and predictability of the 28-day and hospital mortality in severe sepsis patients [16]. In addition, the JAAM DIC diagnostic criteria can detect twice as many cases and require lesser time than the ISTH overt DIC criteria [17]. The APACHE II scoring system, which is a classification system to measure the severity of disease, consists of three factors as follows: age, chronic disease score, and acute physiology score (APS). It has been widely used in predicting outcomes, including sepsis, for critically ill patients [18]. However, because APS is defined as a sum of the worst values of 12 physiological indicators within $24 \mathrm{~h}$ after ICU admission, the APACHE II score may not be useful in the judgment criteria for starting treatment from the viewpoint of its simplicity and promptness. Thus, based on the previous evidence that DIC is simultaneously associated with increasing disease severity, we focused on age, one of the components of the APACHE II score. A previous study demonstrated that aging is accompanied by an increased prothrombotic state [19], suggesting that age may be a key factor involved in the pathophysiology of sepsis-associated DIC, affecting the efficacy of anticoagulant therapy against sepsis.

Therefore, the objective of this study was to examine the age-related differences in the survival benefit of anticoagulant therapy in sepsis, in accordance with the JAAM DIC diagnostic criteria, using the nationwide sepsis registry data set.

\section{Materials And Methods}

\section{Study Design, Setting, and Ethical Approval}

This study is a retrospective sub-analysis of a Sepsis cohort of the prospective multicenter study in the JAAM Focused Outcomes Research in Emergency Care in Acute Respiratory Distress Syndrome, Sepsis and Trauma (FORECAST) study, which is a multicenter nationwide prospective cohort study in Japan [20]. The JAAM FORECAST Sepsis study was conducted from January 2016 to March 2017 and used consecutive samples from 59 intensive-care units (ICUs) in Japan. The study was registered at the University Hospital Medical Information Network Clinical Trial Registry. (UMIN-CTR ID: UMIN000019588). 
This study was approved under the condition that written informed consent was to be obtained from the patient or next of kin by the JAAM and the Ethics Committee of each hospital (JAAM, 2014-01; Hokkaido University Faculty of Medicine, Head institute of the FORECAST group, 014-0307) and was performed in accordance with the Declaration of Helsinki.

\section{Participants}

The JAAM FORECAST Sepsis study enrolled adult patients (> 16 years of age) with severe sepsis and septic shock, based on the Sepsis-2 criteria published in 2003 [21], who had been admitted to the ICU when they met the following inclusion criteria: (i) patients suspected to have or were diagnosed with newonset infection based on the history of the present illness (ii) patients who met $\geq 2$ systemic inflammatory response syndrome criteria and (iii) patients who had at least one organ dysfunction. The inclusion criteria (iii) was further defined by the following criteria: systolic blood pressure $<90 \mathrm{mmHg}$ or mean arterial pressure $<65 \mathrm{mmHg}$ or decreased blood pressure $>40 \mathrm{mmHg}$ from the baseline; serum creatinine $>2.0 \mathrm{mg} / \mathrm{dL}$ or diuresis $<0.5 \mathrm{~mL} / \mathrm{kg} / \mathrm{h}$; total bilirubin $>2.0 \mathrm{mg} / \mathrm{dL}$; platelet counts $<100 \times 10^{9} / \mathrm{L}$; lactate $>2 \mathrm{mmol} / \mathrm{L}$; prothrombin time International Normalized Ratio (INR) $>1.5$; arterial hypoxemia $(\mathrm{PaO} 2 / \mathrm{FiO} 2)<200$ with pneumonia or $\mathrm{PaO} 2 / \mathrm{FiO} 2<250$ without pneumonia. Patients on end-of-life care or resuscitated from cardiac arrest at the time of the diagnosis of severe sepsis were excluded. Patients with substantial missing data were also excluded from the analysis.

Participants were divided into two groups: the anticoagulant group and the non-anticoagulant group. The anticoagulant group consisted of patients who received anticoagulant therapy, defined as the administration of antithrombin, recombinant human thrombomodulin and their combination, based on the Japanese Clinical Practice Guidelines for Management of Sepsis and Septic Shock 2020 [22]. The non-anticoagulant group consisted of patients who received neither antithrombin nor recombinant human thrombomodulin. There was no pre-determined definitive protocol for the indication of anticoagulant therapy, and it was applied at the discretion of the participating physicians based on the treatment policies of each hospital. The standard dosage and administration for antithrombin for sepsisinduced DIC in Japan is $1,500 \mathrm{U} /$ day or $30 \mathrm{U} / \mathrm{kg} /$ day, 3-5 days, and that of recombinant human thrombomodulin is $380 \mathrm{U} / \mathrm{kg}, 6$ days, respectively.

\section{Definitions}

Systemic inflammatory response syndrome (SIRS), sepsis, severe sepsis and septic shock were defined based on the American College of Chest Physicians/Society of Critical Care Medicine consensus conference (Sepsis-1) [23] published in 1992 and its revised version (Sepsis-2) published in 2003 [21]. The disease severity of the patients was assessed according to the APACHE II score [15]. Organ dysfunction was evaluated based on the Sequential Organ Failure Assessment (SOFA) score [24]. The Charlson index was adopted for the assessment of the baseline comorbidities [25]. The DIC diagnosis was made based on the JAAM DIC scoring system using prothrombin time INR as a substitute for prothrombin time ratio (Supplementary Table 1. See Additional file 1) [26]. 


\section{Data Collection}

An electronic data capture system was developed for use in this FORECAST study. The data, which were compiled by the FORECAST investigators, were obtained from the FORECAST database. Patient information included the baseline characteristics, various comorbidities, activities of daily living (ADL), suspected sites of infection, organ dysfunction, sepsis-related severity scores, and therapeutic interventions. In addition, we obtained data on compliance with established sepsis care protocols, such as the measurement of serum lactate within $3 \mathrm{~h}$. The primary outcome was in-hospital all-cause mortality. The SOFA score at $72 \mathrm{~h}$ after admission was recorded as the secondary outcome.

\section{Statistical Analyses}

All baseline clinical and demographical characteristics were expressed as medians and inter-quantile ranges for continuous variables whereas numbers and percentages for categorical variables. For comparisons of these characteristics between the patients with and without anticoagulants, the MannWhitney $\mathrm{U}$ test, and the chi-square test were used for the continuous variables and the categorical variables, respectively.

To examine whether the effect of the anticoagulants on the hospital mortality was modified by the DIC score, we performed a multivariable Cox proportional-hazard regression model including a cross-product term between the anticoagulants' variable and DIC score in addition to the main effect terms as the explanatory variables. The non-linear effect of the DIC score was assessed using a restricted-cubic-spline method with knot, $k=3$. Furthermore, we assessed the effect of the age of the patients as the effect modifier using a similar regression model including three- and two-way cross-product terms between the variables indicating the anticoagulants, the DIC score, and age. Moreover, similar analyses were performed for $72 \mathrm{~h}$ SOFA as the explained variable using the multivariable non-linear regression models. These regression models were adjusted for all covariates described in Table 1. We confirmed that these regression models were not overfitting using the bootstrap validation (index-corrected calibration slopes were around 0.8). The missing values were imputed using a multiple imputation method. 
Table 1

Baseline clinical characteristics of the patients with and without anticoagulant therapy

$\begin{array}{ll}\text { Non-anticoagulant group } & \text { Anticoagulant group } \\ \mathrm{N}=809 & \mathrm{~N}=331\end{array}$

Patient characteristics

\begin{tabular}{llll|} 
Age & $73(64-82)$ & $72(64-81)$ & 0.609 \\
\hline Sex Female/Male & $38.7 / 61.3(313 / 496)$ & $41.7 / 58.3(138 / 193)$ & 0.347 \\
\hline Preexisting condition & & & \\
\hline Charlson comorbidity index & $1(0-2)$ & $1(0-2)$ & 0.017 \\
\hline ADL Dependent/Independent & $25.1 / 74.9(203 / 606)$ & $21.2 / 78.8(70 / 260)$ & 0.164 \\
\hline Malignant disease, No/Yes & $87.4 / 12.6(707 / 102)$ & $(276 / 55)$ & 0.075 \\
\hline Severe liver disease, No/Yes & $98.1 / 1.9(794 / 15)$ & $97.0 / 3.0(321 / 10)$ & 0.222 \\
\hline Prescribed anticoagulants, No/Yes & $90.6 / 9.4(733 / 76)$ & $91.2 / 8.8(302 / 29)$ & 0.737 \\
\hline IIIness severity & & & $<0.001$ \\
\hline APACHE II score & $21(16-28)$ & $27(20-33)$ & $<0.001$ \\
\hline SOFA score & $8(5-11)$ & $10(7-13)$ & 0.176 \\
\hline SIRS score & $3(2-4)$ & $3(2-4)$ & $<0.001$ \\
\hline ISTH DIC score & $2(1-4)$ & $4(3-5)$ & $<0.001$ \\
\hline JAAM DIC score & $3(2-5)$ & $5(4-6)$ & $<0.001$ \\
\hline Septic shock, No/Yes & $44.5 / 55.5(360 / 449)$ & $20.5 / 79.5(68 / 263)$ & 0.012 \\
\hline Blood culture, Negative/Positive & $43.4 / 56.6(349 / 455)$ & $35.3 / 64.7(117 / 214)$ & $<0.001$ \\
\hline Primary site of infection & & $35.6(118)$ & \\
\hline Abdomen & $21.6(175)$ & $20.5(68)$ & $19.6(65)$ \\
\hline Lung & $35.6(288)$ & & \\
\hline Urinary tract & $18.8(152)$ & & \\
\hline
\end{tabular}

Reported proportion (count) for categorical and median (interquartile range) for continuous variables.

Abbreviations: ADL, activities of daily living; APACHE, Acute Physiology and Chronic Health Evaluation; CNS, central nerve system; CRRT, continuous renal replacement therapy; DIC, disseminated intravascular coagulation; IVIg, intravenous immunoglobulin; ISTH, International Society on Thrombosis \& Haemostasis; JAAM, Japanese Association for Acute Medicine; PMX-DHP, polymyxin B direct hemoperfusion; SIRS, systemic inflammatory response syndrome; SOFA, Sequential Organ Failure Assessment. 


\begin{tabular}{|c|c|c|c|}
\hline & $\begin{array}{l}\text { Non-anticoagulant group } \\
\mathrm{N}=\mathbf{8 0 9}\end{array}$ & $\begin{array}{l}\text { Anticoagulant group } \\
\mathrm{N}=331\end{array}$ & $p$ \\
\hline Skin/Soft tissue & $9.6(78)$ & $10.3(34)$ & \\
\hline Blood stream & $2.1(17)$ & $1.2(4)$ & \\
\hline Bone/Joint & $2.0(16)$ & $1.2(4)$ & \\
\hline CNS & $1.7(14)$ & $2.1(7)$ & \\
\hline Endocardium & $1.4(11)$ & $1.5(5)$ & \\
\hline Implant device & $0.9(7)$ & $0.3(1)$ & \\
\hline Wound & $1.0(8)$ & $0.6(2)$ & \\
\hline Others & $5.3(43)$ & $6.9(23)$ & \\
\hline \multicolumn{4}{|l|}{ Therapeutic interventions } \\
\hline Mechanical ventilation & $52.2 / 47.8(420 / 384)$ & $42.4 / 57.6(140 / 190)$ & 0.003 \\
\hline PMX-DHP & 96.3/3.7 (779/30) & $79.5 / 20.5(263 / 68)$ & $<0.001$ \\
\hline IVIg & $91.1 / 8.9(733 / 72)$ & $54.3 / 45.7(178 / 150)$ & $<0.001$ \\
\hline Protease inhibitor & $95.0 / 5.0(768 / 40)$ & $86.3 / 13.7(283 / 45)$ & $<0.001$ \\
\hline CRRT & $82.5 / 17.5(664 / 141)$ & $51.7 / 48.3(171 / 160)$ & $<0.001$ \\
\hline Corticosteroids & $77.4 / 22.6(625 / 182)$ & $51.2 / 48.8(169 / 161)$ & $<0.001$ \\
\hline Noradrenalin & $41.1 / 58.9(332 / 475)$ & $19.0 / 81.0(63 / 268)$ & $<0.001$ \\
\hline Enteral nutrition & $55.8 / 44.2(450 / 357)$ & 49.8/50.2 (164/165) & 0.070 \\
\hline \multicolumn{4}{|c|}{ Reported proportion (count) for categorical and median (interquartile range) for continuous variables. } \\
\hline \multicolumn{4}{|c|}{$\begin{array}{l}\text { Abbreviations: ADL, activities of daily living; APACHE, Acute Physiology and Chronic Health } \\
\text { Evaluation; CNS, central nerve system; CRRT, continuous renal replacement therapy; DIC, disseminated } \\
\text { intravascular coagulation; IVIg, intravenous immunoglobulin; ISTH, International Society on } \\
\text { Thrombosis \& Haemostasis; JAAM, Japanese Association for Acute Medicine; PMX-DHP, polymyxin B } \\
\text { direct hemoperfusion; SIRS, systemic inflammatory response syndrome; SOFA, Sequential Organ } \\
\text { Failure Assessment. }\end{array}$} \\
\hline
\end{tabular}

All hypothesis tests were conducted with a two-sided $5 \%$ significance level using the R software (https://cran.r-project.org/).

\section{Results}

\section{Study Population}


In total, 1,184 consecutive patients fulfilling the inclusion criteria were registered during the study period in the FORECAST Sepsis cohort. Six registered patients who had missing values exceeding the threshold (>170) were detected by a one-sample robust regression with an $\mathrm{M}$ estimator. Thirty-eight patients were also excluded due to missing information on the administration of antithrombin and recombinant human thrombomodulin. We analyzed the final cohort of 1140 patients in the present study. The anticoagulant group comprised 331 patients (antithrombin, 89 patients; recombinant human thrombomodulin, 100; and their combination, 142), and the non-anticoagulant group comprised 809 patients (Fig. 1).

Table 1 shows baseline clinical patient characteristics and therapeutic interventions in the patients with or without anticoagulant treatments. Patient characteristics such as the age and sex were similar between the two groups. The Charlson comorbidity index, APACHE II, SOFA, and DIC scores were significantly higher in the anticoagulant group than those in the non-anticoagulant group. In addition, the anticoagulant group showed higher rates of developing septic shock and positive blood culture than the non-anticoagulant group. The most common sites of infection were lung in the non-anticoagulant group and abdomen in the anticoagulant group, respectively, and these rates differed between the two groups. The rates of concomitant therapeutic interventions were also different between the two groups.

\section{The Effect of Anticoagulant Therapy on Hospital Mortality}

The cumulative survival probability that was obtained in overall patients included in this study is shown in Fig. 2. The hospital mortality rate was $30.2 \%(100 / 331)$ in the anticoagulant group and $20.5 \%$ $(166 / 809)$ in the non-anticoagulant group. The multivariable regression model showed that the hazard ratio (anticoagulant/non-anticoagulant) of hospital mortality was 0.910 (upper 0.665 , lower $1.245, p=$ $0.554)$.

We performed the multivariate Cox proportional hazard regression model that included a two-way interaction to identify the effect modification of anticoagulant therapy by DIC score (Fig. 3A). Neither anticoagulant therapy nor DIC score showed significant interaction with hospital mortality $(p=0.773$ and $p=0.777$, respectively). The $p$-value for the two-way interaction was 0.687 . The multivariate Cox proportional hazard regression model including the three-way interaction term between the previous two ones (anticoagulant therapy and DIC score) and age, which may affect the therapeutic effect, showed that the increases in the risk were suppressed in the population with higher DIC scores in the anticoagulant group in patients aged 60 to 70 years. In sepsis patients aged 50 years, the risk in the nonanticoagulant group tended to increase concomitantly with increases in the DIC score in the low score range (DIC score $<4$ ), while there was no increase in the risk in the high score range (DIC score $\geq 4$ ) and a favorable association of anti-coagulant therapy on hospital mortality was not found. In patients aged 80 years, the non-anticoagulant group indicated a certain risk regardless of the DIC score and anti-coagulant therapy showed no beneficial effect on the decrease in risk of hospital mortality. Importantly, anticoagulant therapy in the lower DIC score range increased the risk hazard in patients aged 50 to 60 years. Global $p$-values for the age and three-way interaction were 0.041 and 0.055 , respectively (Fig. 3B).

\section{SOFA score $72 \mathrm{~h}$ after admission according to the DIC score}


The multivariate linear regression model was used to elucidate the effect modification of anticoagulant therapy on organ dysfunction by the DIC score (Fig. 4). The risk hazard of the SOFA score $72 \mathrm{~h}$ after admission in the non-anticoagulant group increased concomitantly with the increase in the DIC score. The anticoagulant therapy showed no favorable association with the SOFA score. In addition, when we considered the population with lower DIC scores, a higher risk of increase in the SOFA score was found in the anticoagulant group (Fig. 4A). We assessed the age-related effect of anticoagulant therapy on the SOFA score, as well as hospital mortality (Fig. 4B). The anticoagulant therapy did not improve the SOFA score at any age, and the therapy tended to deteriorate SOFA scores at the low DIC score range.

\section{Discussion}

We investigated the age-related differences in the survival benefit of anticoagulant therapy in sepsis in accordance with the JAAM DIC diagnostic criteria. The results of this study indicated that anticoagulant therapy was associated with better outcomes consistent with a higher DIC score in patients aged around 60 to 70 years. Moreover, anticoagulant therapy in septic patients with low DIC scores may be associated with the deterioration of organ dysfunction and poor outcomes.

Previous studies demonstrated that septic patients with DIC were associated with an increased prevalence of multiple organ dysfunction and that the mortality rate of such patients was significantly higher than that of non-DIC patients [16,27], suggesting that the development of DIC in sepsis is a poor prognosis factor. A recent nationwide multicenter retrospective cohort study suggested that screening for DIC was associated with a survival benefit in patients with sepsis [28]. These results imply that the evaluation and the subsequent intervention for coagulofibrinolytic changes related to sepsis may contribute to the improvement of outcomes in patients with sepsis. To date, however, there has not been clear evidence of treating sepsis-induced coagulopathy with improved outcomes of patients with sepsis.

The important point is that most of the RCTs that evaluated the effect of anticoagulant therapy have targeted patients with "sepsis." Although the KyberSept trial, a mega-RCT, demonstrated that antithrombin treatment did not affect the 28-day mortality in adult patients with sepsis and septic shock [5], its posthoc analysis suggested that antithrombin therapy was associated with a significant reduction in mortality only in the patients with DIC but not in the non-DIC patients [29]. These results indicate the importance of selecting the target population for anticoagulant therapy against sepsis. Studies showing that the optimal target for anticoagulant therapy is the patient population fulfilling three factors of sepsis, DIC and high disease severity have recently been published [13, 14, 30-32].

A recent global analysis demonstrated that 1 in 5 deaths around the world is caused by sepsis leading to 11 million deaths annually worldwide, which is more than those killed by cancer [33]. Elderly people make up the major population of patients with sepsis and mortality increases at more advanced ages [34, 35]. Although it is well known that advancing age is associated with increased coagulation, this propensity of deteriorating coagulation disorder during sepsis remains unknown [34]. The innate immune cells such as neutrophils and macrophages show age-related functional alteration, resulting in a diminished ability to 
rapidly respond to foreign pathogens [36]. In addition to the innate immune system, an attenuation of the adaptive immune functions in old age has been confirmed [37]. A prolonged inflammatory response in aged patients has been noted, as high levels of inflammatory cytokines have been found [38-41]. The interaction between the effect of anticoagulant therapy and the DIC score in patients aged 80 years, which were found in our study may support these previous studies. In other words, the onset of sepsis itself might determine the outcome regardless of the severity of DIC in the elderly. In contrast, patients aged 50 years showed a different interaction between the anticoagulant therapy and the DIC score. The relatively young septic patients are expected to respond well to the treatment of underlying infection, followed by the modulation of dysregulated inflammatory and couagulofibrinolytic reactions. Therefore, it is presumed that the development of DIC in the relatively young septic patients may have little effect on the outcome, while the detailed mechanisms remain unclear.

The present study failed to show the beneficial effect of anticoagulant therapy on the SOFA score of the population of any age. However, it does not mean that organ dysfunction cannot be improved by anticoagulant therapy because the SOFA scores were evaluated relatively early (at $72 \mathrm{~h}$ after admission). As anticoagulant therapy improved hospital mortality in certain ages, it may improve the SOFA scores later than $72 \mathrm{~h}$ after admission. Our results should be considered when setting up the study designs of future RCTs to validate the effects of anticoagulant therapy against sepsis.

The main pathophysiology of DIC is uncontrolled thrombin generation, leading to ischemic organ dysfunction due to microvascular thrombosis, which is detrimental in the context of pathology. In contrast, the concept that microvascular thrombosis produced by innate immunity is a physiological process to maintain body homeostasis has come to be known as immunothrombosis [3, 42]. From these perspectives, those who could be at an advantage when immunothrombosis is considered, namely, nonDIC patients, and they should not be treated by anticoagulant therapy. The harmful effects of anticoagulant therapy against non-DIC patients were confirmed in the present study (Figs. 3 and 4).

The current study is associated with several limitations. First, although the present data set was prospectively collected, causal relationships could not be defined because of the study's retrospective design. Second, this study did not assess the dosage and duration of anticoagulant agents. Additionally, this study was unable to evaluate the effects of individual anticoagulant agents or combination therapy because we defined anticoagulant therapy as the administration of antithrombin, recombinant human thrombomodulin, and their combination. Third, the present study data set did not include the information on the adverse effects, including serious bleeding complications associated with anticoagulant therapy, which can cause unfavorable outcomes. Fourth, data elements required to control potential confounders might result in biased effect estimates. Finally, the study being conducted in a single country may limit the generalizability of the obtained results.

\section{Conclusions}


In conclusion, anticoagulant therapy, the administration of antithrombin, recombinant human thrombomodulin and their combination, showed a beneficial effect on hospital mortality according to a higher JAAM DIC score in septic patients aged around 60 to 70 years. Anticoagulant therapy, however, was not associated with a better outcome in relatively young (50 years) and older (80 years) patients. In addition, anticoagulant therapy in patients with low DIC scores were associated with the deterioration of organ dysfunction and poor outcome, which may be caused by the destruction of the physiological hypercoagulative state, immunothrombosis. We suggest setting the inclusion criteria of future RCTs to examine the effects of anticoagulant therapy against sepsis based on the results obtained from the present study.

\section{Abbreviations}

$\mathrm{ADL}$

Activities of daily living; APACHE:Acute Physiology and Chronic Health Evaluation; APS:Acute physiology score; CNS:Central nerve system; CRRT:Continuous renal replacement therapy; DIC:Disseminated intravascular coagulation; FORECAST:Focused Outcomes Research in Emergency Care in Acute Respiratory Distress Syndrome, Sepsis and Trauma; ICUs:Intensive care units; INR:International Normalized Ratio; ISTH:International Society on Thrombosis and Haemostasis; IVIg:Intravenous immunoglobulin; JAAM:Japanese Association for Acute Medicine; PMX-DHP:Polymyxin B direct hemoperfusion; RCTs:large-scale randomized clinical trials; SIRS:Systemic inflammatory response syndrome; SOFA:Sequential organ failure assessment; UMIN-CTR:University Hospital Medical Information Network Clinical Trial Registry;

\section{Declarations}

\section{ETHICS APPROVAL AND CONSENT TO PARTICIPATE}

This study was approved under the condition that written informed consent was to be obtained from the patient or next of kin by the JAAM and the Ethics Committee of each hospital (JAAM, 2014-01; Hokkaido University Faculty of Medicine, Head institute of the FORECAST group, 014-0307) and was performed in accordance with the Declaration of Helsinki.

\section{CONSENT FOR PUBLICATION}

Not applicable.

\section{AVAILABILITY OF DATA AND MATERIALS}

The datasets during and/or analyzed during the current study available from the corresponding author on reasonable request. 
This study was supported by the Japanese Association for Acute Medicine (JAAM, 2014-01). None of the authors declare any conflicts of interest in connection with the submitted article.

Gando S reported receiving personal fees from Grifols outside the submitted work. Shiraishi A reported receiving personal fees from CSL Behring outside the submitted work. The other authors have no conflict of interest to declare.

\section{FUNDING}

This study was supported by the JAAM.

\section{AUTHORS' CONTRIBUTIONS}

TW conceived of and designed this study, contributed to analysis, and interpretation of the data, and was responsible for drafting, editing, and submission of the manuscript. KY contributed to the acquisition of data, interpreted the data, and revised the manuscript for important intellectual content. DK played a significant role in the analysis of the data and helped to draft the manuscript. AS contributed to the acquisition of data, conducted data cleaning, interpreted the data, and revised the manuscript for important intellectual content. SG had a significant influence on the interpretation of the data and critical appraisal of the manuscript. The other authors contributed to the design of the study, organization of data collection, drafting of database charts and scientific discussion of all of the process of the study as well as reviewed the manuscript and registered the patients. All authors approved the final version of the manuscript.

\section{ACKNOWLEDGEMENTS}

The JAAM FORECAST Study Group thanks Shuta Fukuda for his special assistance in completing the study. We would like to thank Editage (www.editage.com) for English language editing.

Investigators of the JAAM FORECAST Study Group: Osaka City University Hospital (Yasumitsu Mizobata); National Hospital Organization Sendai Medical Center (Yasuo Yamada); Saitama Medical University Saitama Medical Center (Satoru Sugiyama); Fukui Prefectural Hospital (Hiroshi Ishida); Sapporo Medical University (Eichi Narimatsu); Fukuyama City Hospital (Koji Miyasho); Hiratsuka City Hospital (Toshio Kanai); Saiseikai Utsunomiya Hospital (Satoru Miyatake); Japanese Red Cross Society Kyoto Daini Hospital (Ryouji liduka); Shinsyu University School of Medicine (Hiroshi Imamura); Rinku General Medical Center (Yasuaki Mizushima); Subaru Health Insurance Society Ota Memorial Hospital (Yoshitake Sato); Saitama Medical University International Medical Center (Manabu Nemoto); Aomori Prefectural Central Hospital (Hiroyuki Hanada); National Hospital Organization Hokkaido Medical Center (Yasuo Shichinohe); Saga-ken Medical Centre Koseikan (Kenji Hirahara); Hachinohe City Hospital (Akihide Kon); Juntendo University Nerima Hospital (Manabu Sugita); Kawaguchi Municipal Medical Center (Yasutaka Naoe); Kakogawa West City Hospital (Manabu Kirita); Osaka National Hospital (Daikai Sadamitsu); Kouseiren Takaoka Hospital (Masahiro Yoshida), Hokkaido University Graduate School of Medicine (Takeshi Wada); Kameda Medical Center (Atsushi Shiraishi); Sapporo Higashi Tokushukai Hospital (Satoshi 
Gando); Osaka Medical College (Kazuma Yamakawa); Keio University School of Medicine (Seitaro Fujishima, Junichi Sasaki); National Defence Medical College (Daizoh Saitoh); Tohoku University Graduate School of Medicine (Shigeki Kushimoto), Osaka University Graduate School of Medicine (Hiroshi Ogura); Tsukuba Memorial Hospital (Toshikazu Abe); University of Occupational and Environmental Health (Toshihiko Mayumi); Kobe University Graduate School of Medicine (Joji Kotani); Aichi Medical University Hospital (Naoshi Takeyama); Yamaguchi University Hospital (Ryosuke Tsuruta); Kawasaki Municipal Hospital (Kiyotsugu Takuma); Kurume University (Norio Yamashita); Aizu Chuo Hospital (Shin-ichiro Shiraishi); Teikyo University School of Medicine (Hiroto Ikeda); Kawasaki Medical School (Yasukazu Shiino); Kyorin University School of Medicine (Takehiko Tarui); Chiba University Graduate School of Medicine (Takaaki Nakada); St. Luke's International Hospital (Toru Hifumi); Kitakyushu City Yahata Hospital (Kohji Okamoto); Saga University Hospital (Yuichiro Sakamoto); Center Hospital of the National Center for Global Health and Medicine (Akiyoshi Hagiwara); Nippon Medical School (Tomohiko Masuno); Community Healthcare Organization, Chukyo Hospital (Masashi Ueyama); Osaka General Medical Center (Satoshi Fujimi, Yutaka Umemura), Tokyo Medical and Dental University, (Yasuhiro Otomo)

\section{References}

1. Taylor FB Jr, Toh CH, Hoots WK, Wada H, Levi M. Towards definition, clinical and laboratory criteria, and a scoring system for disseminated intravascular coagulation. Thromb Haemost. 2001;86:132730.

2. Bone RC. The pathogenesis of sepsis. Ann Intern Med. 1991;115:457-69.

3. Gando S, Levi M, Toh CH. Disseminated intravascular coagulation. Nature reviews Disease primers. 2016;2:16037.

4. Jaimes F, De La Rosa G, Morales C, Fortich F, Arango C, Aguirre D, Munoz A. Unfractioned heparin for treatment of sepsis: A randomized clinical trial (The HETRASE Study). Crit Care Med. 2009;37:118596.

5. Warren BL, Eid A, Singer P, Pillay SS, Carl P, Novak I, Chalupa P, Atherstone A, Penzes I, Kubler A, et al. Caring for the critically ill patient. High-dose antithrombin III in severe sepsis: a randomized controlled trial. JAMA. 2001;286:1869-78.

6. Ranieri VM, Thompson BT, Barie PS, Dhainaut JF, Douglas IS, Finfer S, Gardlund B, Marshall JC, Rhodes A, Artigas A, et al. Drotrecogin alfa (activated) in adults with septic shock. N Engl J Med. 2012;366:2055-64.

7. Bernard GR, Vincent JL, Laterre PF, LaRosa SP, Dhainaut JF, Lopez-Rodriguez A, Steingrub JS, Garber GE, Helterbrand JD, Ely EW, et al. Efficacy and safety of recombinant human activated protein C for severe sepsis. N Engl J Med. 2001;344:699-709.

8. Abraham E, Laterre PF, Garg R, Levy H, Talwar D, Trzaskoma BL, Francois B, Guy JS, Bruckmann M, Rea-Neto A, et al. Drotrecogin alfa (activated) for adults with severe sepsis and a low risk of death. N Engl J Med. 2005;353:1332-41. 
9. Abraham E, Reinhart K, Opal S, Demeyer I, Doig C, Rodriguez AL, Beale R, Svoboda P, Laterre PF, Simon S, et al. Efficacy and safety of tifacogin (recombinant tissue factor pathway inhibitor) in severe sepsis: a randomized controlled trial. JAMA. 2003;290:238-47.

10. Wunderink RG, Laterre PF, Francois B, Perrotin D, Artigas A, Vidal LO, Lobo SM, Juan JS, Hwang SC, Dugernier $\mathrm{T}$, et al. Recombinant tissue factor pathway inhibitor in severe community-acquired pneumonia: a randomized trial. Am J Respir Crit Care Med. 2011;183:1561-68.

11. Vincent JL, Francois B, Zabolotskikh I, Daga MK, Lascarrou JB, Kirov MY, Pettila V, Wittebole X, Meziani F, Mercier E, et al. Effect of a Recombinant Human Soluble Thrombomodulin on Mortality in Patients With Sepsis-Associated Coagulopathy: The SCARLET Randomized Clinical Trial. JAMA. 2019;321:1993-2002.

12. Grimaldi D, Vincent JL. Clinical trial research in focus: rethinking trials in sepsis. The Lancet Respiratory medicine. 2017;5:610-1.

13. Umemura Y, Yamakawa K. Optimal patient selection for anticoagulant therapy in sepsis: an evidencebased proposal from Japan. J Thromb Haemost. 2018;16:462-4.

14. Yamakawa K, Gando S, Ogura H, Umemura Y, Kabata D, Shintani A, Shiraishi A, Saitoh D, Fujishima S, Mayumi T, et al. Identifying sepsis populations benefitting from anticoagulant therapy:A Prospective Cohort Study Incorporating a Restricted Cubic Spline Regression Model. Thromb Haemost. 2019;119:1740-51.

15. Knaus WA, Draper EA, Wagner DP, Zimmerman JE. APACHE II: a severity of disease classification system. Crit Care Med. 1985;13:818-29.

16. Gando S, Saitoh D, Ogura H, Fujishima S, Mayumi T, Araki T, Ikeda H, Kotani J, Kushimoto S, Miki Y, et al. A multicenter, prospective validation study of the Japanese Association for Acute Medicine disseminated intravascular coagulation scoring system in patients with severe sepsis. Crit Care. 2013;17:R111.

17. Iba T, Umemura Y, Watanabe E, Wada T, Hayashida K, Kushimoto S. Diagnosis of sepsis-induced disseminated intravascular coagulation and coagulopathy. Acute Med Surg. 2019;6:223-32.

18. Delibegovic S, Markovic D, Hodzic S. APACHE II scoring system is superior in the prediction of the outcome in critically ill patients with perforative peritonitis. Med Arh. 2011;65:82-5.

19. Wilkerson WR, Sane DC. Aging and thrombosis. Semin Thromb Hemost. 2002;28:555-68.

20. Abe T, Ogura H, Shiraishi A, Kushimoto S, Saitoh D, Fujishima S, Mayumi T, Shiino Y, Nakada T, Tarui T, et al. Characteristics, management, and in-hospital mortality among patients with severe sepsis in intensive care units in Japan: the FORECAST study. Crit Care. 2018;22:322.

21. Levy MM, Fink MP, Marshall JC, Abraham E, Angus D, Cook D, Cohen J, Opal SM, Vincent JL, Ramsay G. 2001 SCCM/ESICM/ACCP/ATS/SIS International Sepsis Definitions Conference. Intensive Care Med. 2003;29:530-8.

22. Nishida O, Ogura H, Egi M, Fujishima S, Hayashi Y, Iba T, Imaizumi H, Inoue S, Kakihana Y, Kotani J,et al. The Japanese Clinical Practice Guidelines for Management of Sepsis and Septic Shock 2016 (JSSCG 2016). J Intensive Care. 2018;6:7. 
23. American College of Chest Physicians/Society of Critical Care Medicine Consensus Conference: definitions for sepsis and organ failure and guidelines for the use of innovative therapies in sepsis. Crit Care Med. 1992;20:864 - 74.

24. Vincent JL, Moreno R, Takala J, Willatts S, De Mendonca A, Bruining H, Reinhart CK, Suter PM, Thijs LG. The SOFA (Sepsis-related Organ Failure Assessment) score to describe organ dysfunction/failure. On behalf of the Working Group on Sepsis-Related Problems of the European Society of Intensive Care Medicine. Intensive Care Med. 1996;22:707-10.

25. Charlson ME, Pompei P, Ales KL, MacKenzie CR. A new method of classifying prognostic comorbidity in longitudinal studies: development and validation. J Chronic Dis. 1987;40:373-83.

26. Gando S, Saitoh D, Ogura H, Mayumi T, Koseki K, Ikeda T, Ishikura H, Iba T, Ueyama M, Eguchi Y, et al. Natural history of disseminated intravascular coagulation diagnosed based on the newly established diagnostic criteria for critically ill patients: results of a multicenter, prospective survey. Crit Care Med. 2008;36:145-50.

27. Gando S, Shiraishi A, Yamakawa K, Ogura H, Saitoh D, Fujishima S, Mayumi T, Kushimoto S, Abe T, Shiino $Y$, et al. Role of disseminated intravascular coagulation in severe sepsis. Thromb Res. 2019;178:182-8.

28. Umemura Y, Yamakawa K, Hayakawa M, Hamasaki T, Fujimi S. Screening itself for disseminated intravascular coagulation may reduce mortality in sepsis: A nationwide multicenter registry in Japan. Thromb Res. 2018;161:60-6.

29. Kienast J, Juers M, Wiedermann CJ, Hoffmann JN, Ostermann H, Strauss R, Keinecke HO, Warren BL, Opal SM. Treatment effects of high-dose antithrombin without concomitant heparin in patients with severe sepsis with or without disseminated intravascular coagulation. J Thromb Haemost. 2006;4:90-7.

30. Umemura Y, Yamakawa K, Ogura H, Yuhara H, Fujimi S. Efficacy and safety of anticoagulant therapy in three specific populations with sepsis: a meta-analysis of randomized controlled trials. $\mathrm{J}$ Thromb Haemost. 2016;14:518-30.

31. Yamakawa K, Umemura Y, Hayakawa M, Kudo D, Sanui M, Takahashi H, Yoshikawa Y, Hamasaki T, Fujimi S. Benefit profile of anticoagulant therapy in sepsis: a nationwide multicentre registry in Japan. Crit Care. 2016;20:229.

32. Yoshimura J, Yamakawa K, Ogura H, Umemura Y, Takahashi H, Morikawa M, Inoue Y, Fujimi S, Tanaka $\mathrm{H}$, Hamasaki $\mathrm{T}$, et al. Benefit profile of recombinant human soluble thrombomodulin in sepsis-induced disseminated intravascular coagulation: a multicenter propensity score analysis. Crit Care. 2015;19:78.

33. Rudd KE, Johnson SC, Agesa KM, Shackelford KA, Tsoi D, Kievlan DR, Colombara DV, lkuta KS, Kissoon N, Finfer S, et al. Global, regional, and national sepsis incidence and mortality, 1990-2017: analysis for the Global Burden of Disease Study. Lancet. 2020;395:200-11.

34. Starr ME, Saito H. Sepsis in old age: review of human and animal studies. Aging Dis. 2014;5:126-36. 
35. Angus DC, Linde-Zwirble WT, Lidicker J, Clermont G, Carcillo J, Pinsky MR. Epidemiology of severe sepsis in the United States: analysis of incidence, outcome, and associated costs of care. Crit Care Med. 2001;29:1303-10.

36. Gomez CR, Nomellini V, Faunce DE, Kovacs EJ. Innate immunity and aging. Exp Gerontol. 2008;43:718-28.

37. Weksler ME, Goodhardt M, Szabo P. The effect of age on B cell development and humoral immunity. Springer Semin Immunopathol. 2002;24:35-52.

38. Kale S, Yende S, Kong L, Perkins A, Kellum JA, Newman AB, Vallejo AN, Angus DC. The effects of age on inflammatory and coagulation-fibrinolysis response in patients hospitalized for pneumonia. PLoS One. 2010;5:e13852.

39. Naffaa M, Makhoul BF, Tobia A, Kaplan M, Aronson D, Saliba W, Azzam ZS. Interleukin-6 at discharge predicts all-cause mortality in patients with sepsis. Am J Emerg Med. 2013;31:1361-4.

40. Marik PE, Zaloga GP. The effect of aging on circulating levels of proinflammatory cytokines during septic shock. Norasept II Study Investigators. J Am Geriatr Soc. 2001;49:5-9.

41. Bruunsgaard H, Skinhøj P, Qvist J, Pedersen BK. Elderly humans show prolonged in vivo inflammatory activity during pneumococcal infections. J Infect Dis. 1999;180:551-4.

42. Engelmann B, Massberg $S$. Thrombosis as an intravascular effector of innate immunity. Nature reviews Immunology. 2013;13:34-45.

\section{Figures}




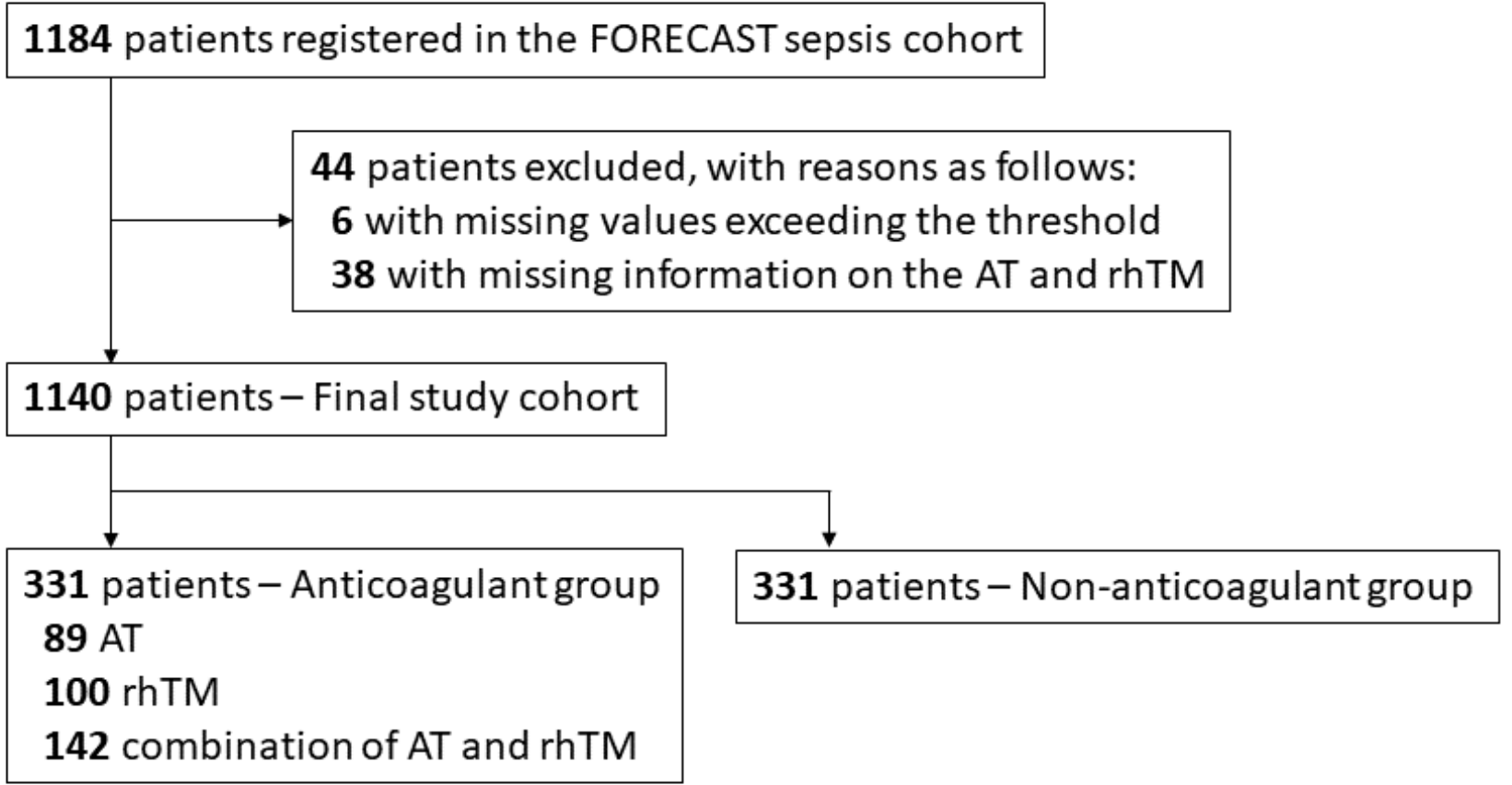

Figure 1

Flow chart of the study population. AT, antithrombin; FORECAST, Focused Outcomes Research in Emergency Care in Acute Respiratory Distress Syndrome, Sepsis and Trauma; rhTM, recombinant human thrombomodulin. 


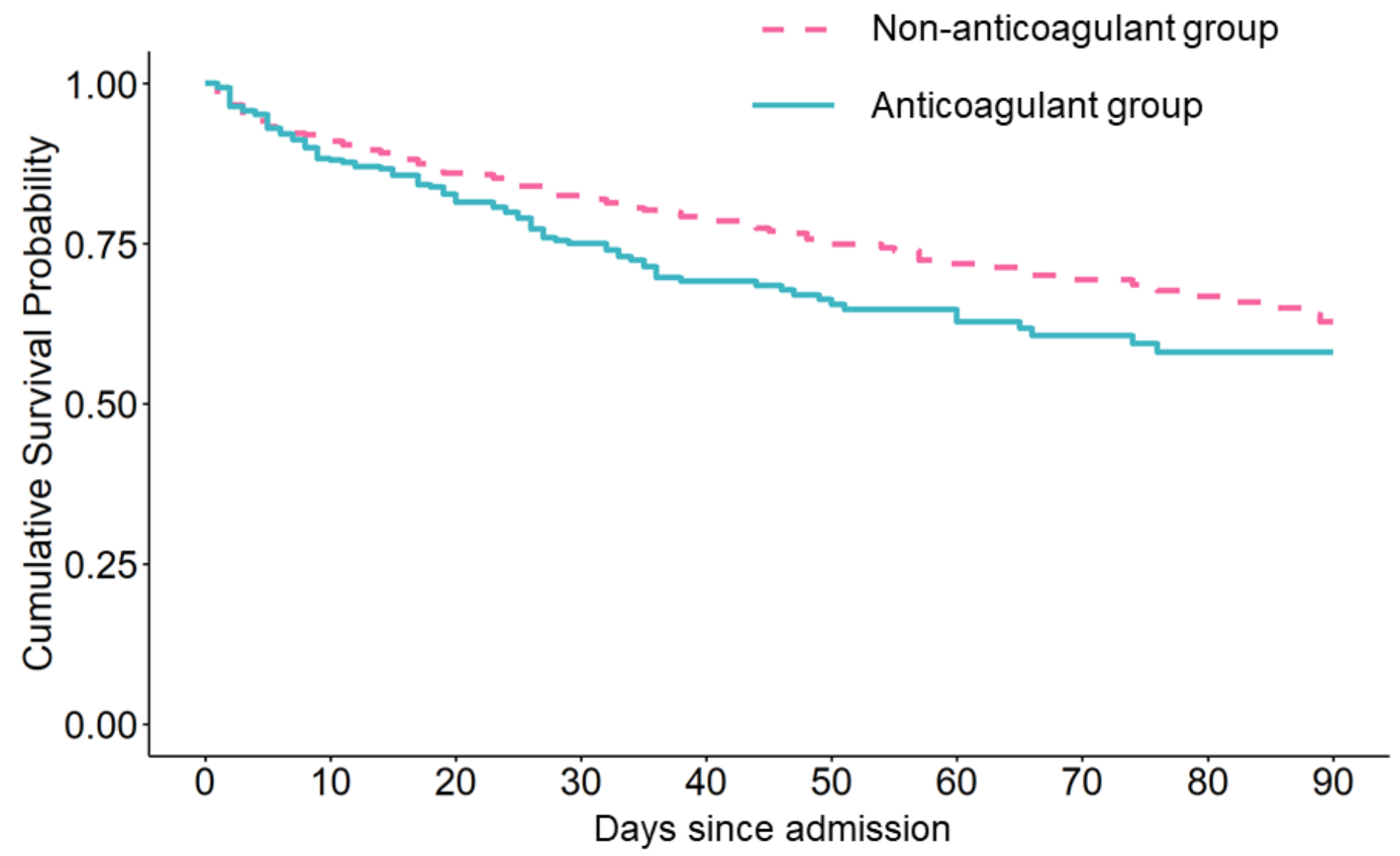

Number at Risk

$\begin{array}{llllllllll}809 & 645 & 449 & 312 & 222 & 169 & 129 & 98 & 74 & 58 \\ 330 & 276 & 213 & 156 & 111 & 86 & 69 & 52 & 37 & 30\end{array}$

Figure 2

The Kaplan-Meier curves during the first 90 days for the cumulative survival of the patients with and without anticoagulant therapy. The blue line represents the anticoagulant group and the dotted red line represents the non-anticoagulant group. 
$=\quad$ Non-anticoagulant group

A

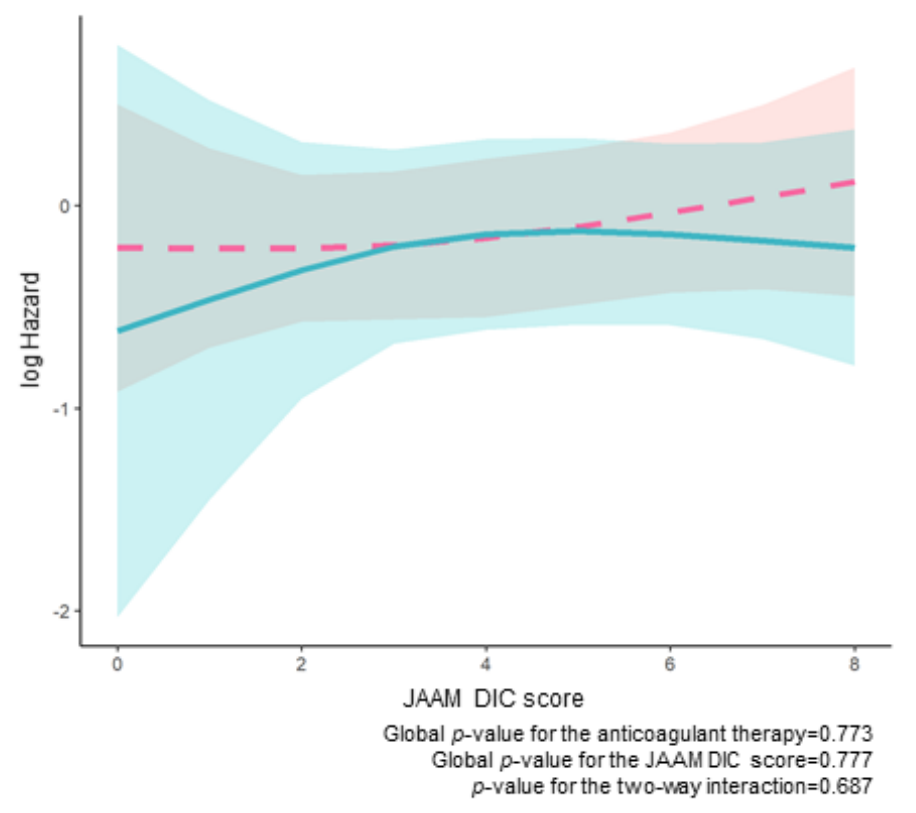

B

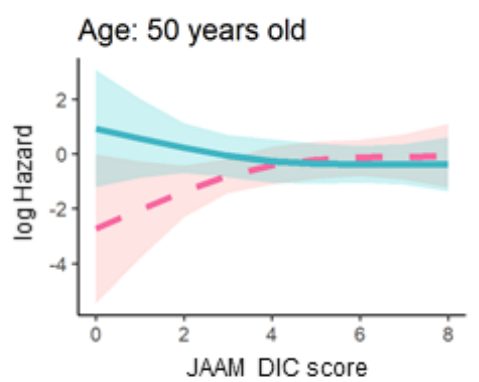

Age: 70 years old
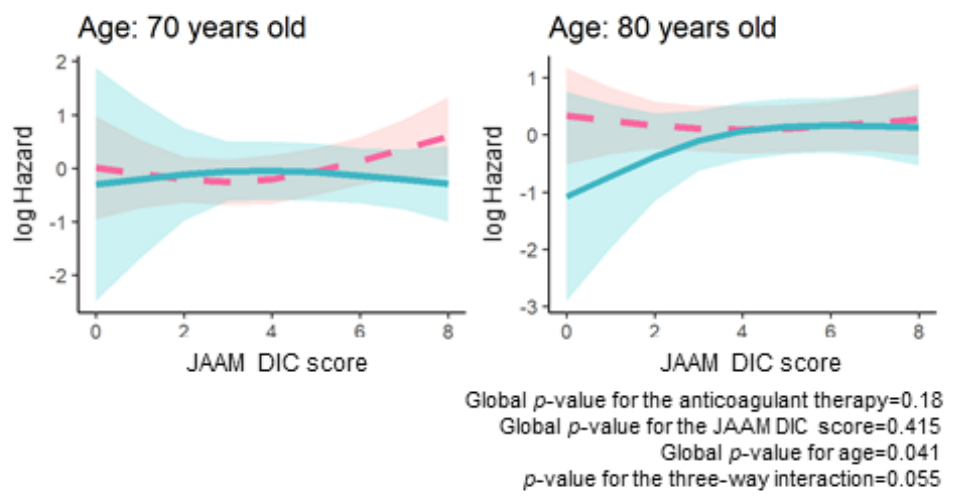

\section{Figure 3}

Regression line of hospital mortality of each treatment group estimated by the Cox proportional hazard regression model. A: Two-way interaction term between the treatment and the JAAM DIC score. B: Threeway interaction term among anticoagulant therapy, the JAAM DIC score, and age. The lines indicate estimated log-transformed relative hazard, and the shaded areas represent $95 \%$ confidence intervals. The solid blue line represents patients in the anticoagulant group, and the dotted red line represents those in the non-anticoagulant group. JAAM, the Japanese Association for Acute Medicine; DIC, disseminated intravascular coagulation. 
$=\quad$ Non-anticoagulant group

A

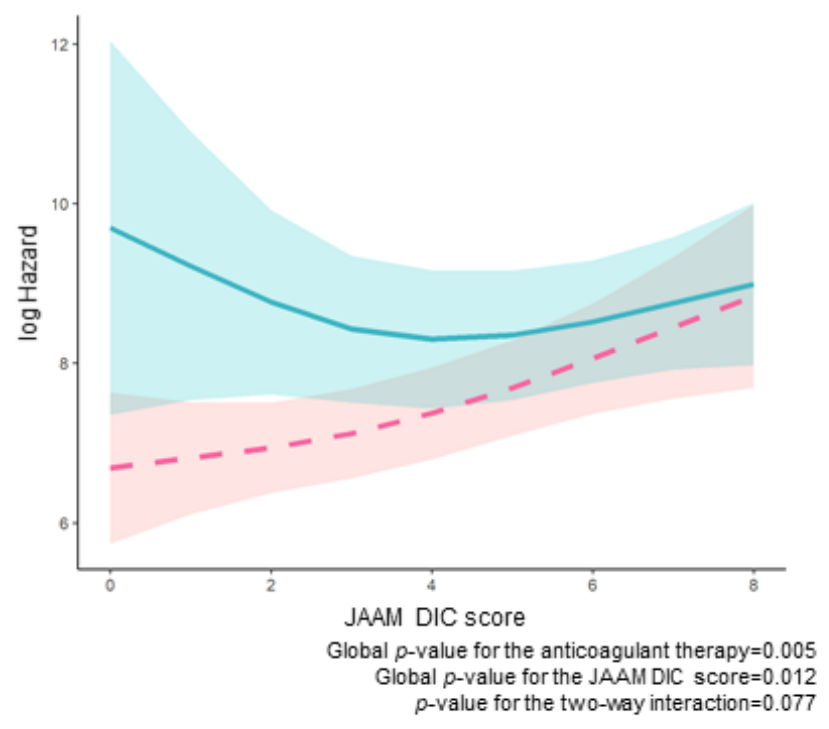
$p$-value for the two-way interaction $=0.077$
B

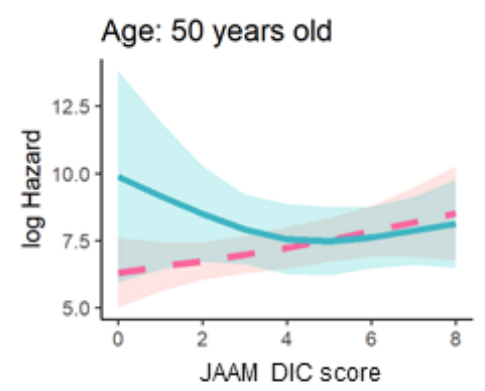

Age: 70 years old

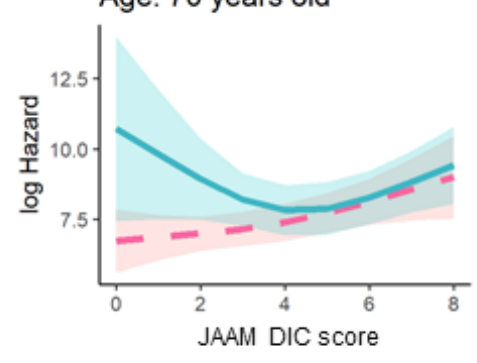

- Anticoagulant group

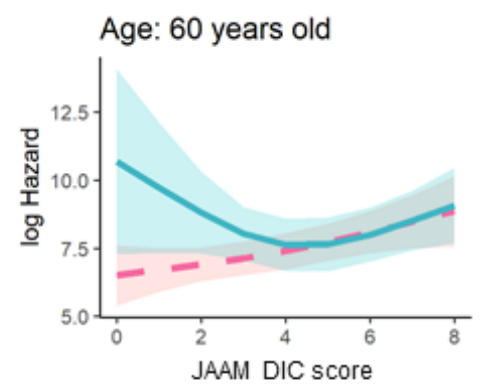

Age: 80 years old

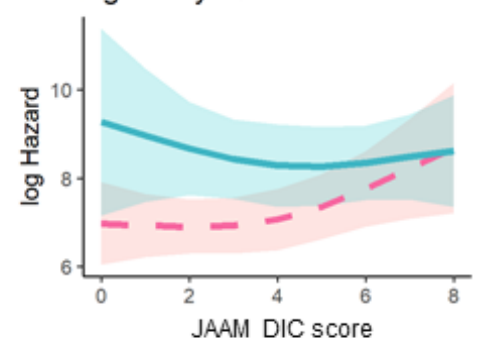

Global $p$-value for the anticoagulant therapy $=0.008$ Global $p$-value for the JAAM DIC score $=0.053$ $p$-value for the three-way interaction $=0.384$

\section{Figure 4}

Regression lines of the SOFA score $72 \mathrm{~h}$ after admission of each treatment group. A: Two-way interaction term between the treatment and the JAAM DIC score. B: Three-way interaction term among anticoagulant therapy, JAAM DIC score, and age. The lines indicate estimated log-transformed relative hazard, and the shaded areas represent $95 \%$ confidence intervals. The solid blue line represents patients in the anticoagulant group, and the dotted red line represents those in the non-anticoagulant group. JAAM, the Japanese Association for Acute Medicine; DIC, disseminated intravascular coagulation.

\section{Supplementary Files}

This is a list of supplementary files associated with this preprint. Click to download.

- Additionalfile1.pdf 